\title{
Correlation between kinetic fragility and Poisson's ratio from analysis of data for soft colloids
}

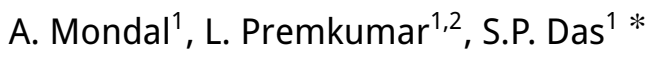 \\ 1 School of Physical Sciences, Jawaharlal Nehru University, New Delhi 110067, India \\ 2 Department of Physics, National Institute of Technology Manipur, Imphal 795004, India
}

Received August 10, 2020, in final form November 10, 2020

\begin{abstract}
We consider the link between fragility and elasticity that follows from the analysis of the data for a set of soft colloid materials consisting of deformable spheres reported by [Mattsson et al., Nature, 2009, 462, 83]. The present work makes a quantitative analysis through an explicit definition for fragility index $m$ in terms of density dependence, extending the corresponding formula of $m$ for molecular systems in terms of temperature dependence. In addition, we fit the data for the high-frequency shear modulus for the respective soft-colloid to a corresponding theoretical expression for the same modulus. This expression for the elastic constant is in terms of the corresponding pair correlation function for the liquid treated as of uniform density. The pair correlation function is adjusted through a proper choice of the parameters for the two body interaction potential for the respective soft-colloid material. The nature of correlation between the fragility and Poisson ratio observed for the soft colloids is qualitatively different, as compared to the same for molecular glasses. The observed link between fragility of a metastable liquid and its elastic coefficients is a manifestation of the effects of structure of the fluid on its dynamics. The present work thus analyses the data on soft colloids and by introducing definitions from statistical mechanics obtains a correlation between kinetic fragility and Poissons's ratio for the soft material.
\end{abstract}

Key words: kinetic fragility, elastic response, relaxation times, glass transition

\section{Introduction}

An instructive plot of the final relaxation time $\tau_{\alpha}$ (on a logarithmic scale) of supercooled liquids was made by Angell [1, 2] with respect to the inverse temperature scaled with $T_{\mathrm{g}}$, i.e., with $x=T_{\mathrm{g}} / T$. For any specific system, the temperature $T_{\mathrm{g}}$ is defined to be the one at which the relaxation time $\tau_{\alpha}$ grows by a chosen order of magnitudes $\mathcal{B}$ (say) over a characteristic short time value. The quantity $\mathcal{B}$ is the same for all materials and generally it is chosen to be 16 [3, 4] in molecular systems. Therefore, the Angell plot, by construction, has all the relaxation curves merging at a single point on the $y$ axis at $y=\mathcal{B}$ and at $x=1$, i.e., for $T=T_{\mathrm{g}}$. Slope of the relaxation time $\tau_{\alpha}$ (on a $\log$ scale) vs scaled temperature $x$ at $T=T_{\mathrm{g}}$ is termed as the fragility index $m$. For fragile liquids, $\tau_{\alpha}$ are very sensitive to temperature changes near $T_{\mathrm{g}}$, and $m$ is large. $\mathrm{SiO}_{2}$ and $o$-terphenyl respectively denote two extreme cases of strong and fragile systems with $m$ values 20 and 81 .

Soft matter [5-8] has constituent elements of much larger size than that of the so-called "hard matter" consisting of atomic size particles. The soft matter is characterized by small values for its elastic moduli. The nature of the glassy dynamics for molecular systems as depicted above is also observed in the relaxation behaviour of soft matter [9-12]. The concentration dependence of relaxation time and elastic properties of deformable colloidal particles have been analyzed at a fixed temperature. The soft colloid materials studied were aqueous suspensions of deformable microgel particles of varying elasticity. The microgel particles consisted of interpenetrated and cross-linked polymer networks of

\footnotetext{
*corresponding author, email address: shankar0359@yahoo.com
} 
poly (N-isopropylacrylamide) and polyacrylic acid. A key thermodynamic property controlling both the elastic and relaxation properties of the soft colloids is the concentration variable $\zeta$. The density of colloid particles, denoted as $\rho$, is determined in terms of polymer concentrations of the microgel suspension. For a system of hard spheres of diameter $d$, the particle concentration $\zeta$ is the same as the packing fraction $\phi$. However, since the microgel particles considered here are deformable, the packing fraction $\phi$ is not simply proportional to the number density. For such systems, we work with the concentration defined as $\zeta=\rho v_{0}$, with $v_{0}$ being the volume of an undeformed sphere. In the hard-sphere limit $v_{0}=\pi d^{3} / 6$, making $\phi \equiv \zeta$. In the model for deformable soft colloids that we present here, physical quantities like relaxation times or shear modulus are plotted as functions of relative concentrations $\left(\zeta / \zeta_{\mathrm{g}}\right)$ or $\left(\zeta / \zeta^{*}\right)$, respectively in terms of concentration at the so-called glass transition point $\left(\zeta_{\mathrm{g}}\right)$ and at a crossover point $\left(\zeta^{*}\right)$. Thus, the role of $v_{0}$ drops out. For simplicity, we are considering a mono-dispersed system, hence size ratio of the particles does not enter, though the present approach can be applied to mixtures as well.

For three different soft colloid materials, logarithms of the relaxation time $\tau_{\alpha}$ were measured using different methods and plotted [13] against relative concentration $\zeta / \zeta_{\mathrm{g}}$ scaled with respect to its value at the so-called glass transition point $\zeta_{\mathrm{g}}$. The latter is defined for soft colloids, in close analogy with $T_{\mathrm{g}}$ for the molecular systems. It is chosen so that the relaxation time $\tau_{\alpha}$ compared to a microscopic scale $\tau_{0}$ grows by a factor $\mathcal{B}$ :

$$
\log _{10}\left[\frac{\tau_{\alpha}\left(\zeta_{\mathrm{g}}\right)}{\tau_{0}}\right]=\mathcal{B}
$$

The result is an Angell-like plot with end points of all three relaxation curves meeting at a single point on the Y-axis, at $x=1$, and $y \equiv \mathcal{B}=5$. Apart from the specific value of $\mathcal{B}$, primarily arising from technical limitations of measuring relaxation times with light scattering, the relative variations within the group of different soft-matters closely resemble those of molecular systems. At a qualitative level, soft matters exhibit similar variations in fragility as that for molecular liquids at fixed volume [1]. The difference in the value of $\mathcal{B}$ for the respective groups also categorizes the glass-forming materials into different classes. For the relaxation times covered in the present Angell plot for soft-colloids, the system is not in a jammed state even at $\zeta_{\mathrm{g}}$, and the Brownian force signifying thermal noise is present.

The fragility $m$ in this case is defined with respect to the concentration variable:

$$
m=\left.\frac{d \log _{10} \tau_{\alpha}}{d\left(\zeta / \zeta_{\mathrm{g}}\right)}\right|_{\zeta=\zeta_{\mathrm{g}}} .
$$

While the relaxation time signifies the liquid-like behaviour, elasticity is a characteristic property related to the rigidity of a system with localized particles. In a disordered fluid state, the elastic behaviour only persists for short times after a stress is applied. The corresponding elastic constant is denoted as $G_{\mathrm{p}}(\zeta)$ [14]. For a set of soft colloids, the plot of $G_{\mathrm{p}}(\zeta)$ vs scaled concentration $\zeta / \zeta^{*}$ is observed to be similar to that of high-frequency shear modulus $G_{\infty}(T)$ vs scaled temperature $T^{*} / T$ for a molecular system [13]. Here, the reference $\zeta^{*}$ is identified as the concentration value over which the stretching exponent for the decay function (of William-Watts form) remains constant. This would signify time temperature superposition. The concentration variable $\zeta$ replaces the temperature in this case. What matters in the present context is its value relative to the scaling density $\zeta / \zeta^{*}$. Concentration dependence of the elastic constant $G_{\mathrm{p}}(\zeta)$ for three different soft colloids [13] is analysed here. From a theoretical perspective [15], high-frequency elastic constants are related to basic interaction of the particles and in case of pairwise additive interaction potentials, results for high-frequency elastic constants are obtained in terms of pair correlation functions $g(r)$ within simplest approximations. Our main focus here is on the structural aspects and we model them in terms of simple two-body interaction potentials.

We focus on two properties of the soft colloids, depicted above, i.e., the fragility and short time elastic response in the soft matter. Relating fragility of a glass-forming system to the interaction potential [1618] has been a topic of much interest. Theoretical studies using landscape paradigm [19, 20] of a many particle system have also been made to further understand this link. Microscopic models for the glassy state, based on either thermodynamic [21-23] or dynamic approach [24-26] simply assume the solid-like properties for the amorphous state. In the thermodynamics based formulation, the metastable states of a disordered solid are described [27, 28] in terms of localized density profiles which also signify vibrational modes [28, 29]. For dynamical models, solid-like behavior for the non-ergodic amorphous state [30] is 
manifested through introduction of Goldstone type modes [24, 31, 32]. Correlations between fragility and elastic properties of glassy systems is also observed in the studies involving the non-ergodicity parameter [33], the bulk and shear moduli [34-37], and the strength of the boson peak [25, 38]. Both of the above two theoretical descriptions of the amorphous state, include the static structure factor which plays a key role in understanding the elastic and dynamic properties.

Reference [13] made an important observation that the data on soft colloids produce a plot similar to what is known as Angell-plot for molecular systems. The present work makes a quantitative analysis of these data through an explicit definition for fragility index $m$ in terms of density dependence, extending the corresponding formula of $m$ for molecular systems in terms of temperature dependence. Thus, we are able to charactaerize each soft colloid material with a value of $m$. We also identify the optimum parameters for interaction for the corresponding solft colloid materials by fitting the experimental data for shear modulus $G$ with the formula obtained using basic statistical mechanics. The bulk modulli $B$ is obtained for the same interaction by evaluating the corresponding formulae for the same that follows from the model used for $G$. Thus, Poisson's ratio $v$ for each material is also calculated. This makes it possible for the present analysis to also study the frequently studied correlation between the fragility $m$ and Poisson's ratio $v$, and this observed correlation is found to be qualitatively contrary to the corresponding result for molecular systems. This new aspect of the "Angell Plot" is something we obtain from our analysis here. In our view, this apparent reversal of the trends in correlations between $v$ and $m$ does not present any contradiction and can also be a consequence of this new type of plots and the corresponding definition of the fragility index as explained above. The paper is organized as follows. In the next section, we discuss the calculation of fragility index from the relaxation curves for the soft colloids. In section III we discuss the theoretical models for short time elastic coefficients in terms of the static correlations. We briefly describe here the input static correlation functions and how the latter is controlled by parameters of the soft sphere interaction potentials. In section IV we discuss the comparison of elastic coefficient data for different colloids and link them to the corresponding fragility parameters. We end the paper with a discussion of results and indicate the limitations of our conclusions.

\section{Angel plot: fragility index}

In the present paper the two basic properties for soft colloid materials on which we focus our analysis, are respectively the fragility index related to the long-time relaxation behaviour and the Poisson ratio related to the high-frequency or equivalently short-time elastic response of the soft matter. In the present section, we discuss the calculation of the first quantity, i.e., the fragility index, using the Angell plot of the relaxation data reported. In the next section we consider the model for high frequency or short-time elastic constants.

\subsection{Angel plot for soft colloids}

To compute the fragility index for a soft colloid material, we analyze the density dependence of the relaxation time $\tau_{\alpha}$ by plotting (on a $\log$ scale) the relaxation time with respect to the concentration $\zeta$, introduced in the previous section. This so-called Angell plot [1, 2, 39, 40] was shown in figure $1 \mathrm{~b}$ of [13] for three different soft colloids, by showing the respective $\log _{10}\left[\tau_{\alpha} / \tau_{0}\right]$ vs the relative concentration $\zeta / \zeta_{\mathrm{g}}$. For discussions of the present work, we refer to the three different soft colloid materials considered here as SC-I, II, and III, respectively. In the Angell plot, the relaxation curves for three respective materials meet at a single point $\zeta / \zeta_{\mathrm{g}} \equiv x=1$. The so-called glass transition point $\zeta_{\mathrm{g}}$ was already defined in the previous section with the relation (1.1) and in the present case the growth in relaxation time is $\mathcal{B}=5$. Assuming that each of the respective relaxation curves for the soft colloids SC-I, II, and III follows a Vogel-Fulcher-like form, the variation of $\tau$ with $\zeta$ is written as

$$
\tau_{\alpha}=\tau_{0} \exp \left[\frac{A \zeta}{\zeta_{\mathrm{K}}-\zeta}\right]
$$

The concentration $\zeta_{\mathrm{K}}$ signifies divergence of the relaxation time and is often identified with the Kauzmann point for a glass-forming material. The fragility index for the soft colloids is defined by a relation similar 
to equation $(1.2)$ for molecular systems.

With the above definition of the fragility, using the relations (1.1), and $(2.1)$ we obtain the relation:

$$
m=\frac{\mathcal{B} \kappa}{\kappa-1} .
$$

$\kappa=\zeta_{\mathrm{K}} / \zeta_{\mathrm{g}}$ is the ratio of respective concentrations $\zeta$ at the so-called Kauzmann point, signifying the divergence of characteristic relaxation time, to the value of $\zeta$ at the glass transition point. Equation (2.1) for the relaxation time can now be presented in the form:

$$
\log _{10}\left[\frac{\tau_{\alpha}(x)}{\tau_{0}}\right]=\mathcal{B} x \frac{\kappa-1}{\kappa-x} .
$$

For a chosen $\mathcal{B}$ characterizing the Angell plot, the parameter $\kappa$ attains a specific value corresponding to each relaxation curve, to obtain the $\tau_{\alpha}(x)$ vs $x$ behaviour for the respective soft-colloid materials in the Angell plot. Using $\kappa$ in the relation (2.2) we obtain the fragility of the corresponding material. The fragility also depends on the chosen value for the constant $\mathcal{B}$. For soft colloids this number is generally smaller than that for a molecular liquid.

\subsection{Fragility index for the soft colloids}

The relaxation times data for the three samples as obtained from figure $2 b$ of [13] are shown in table 1 . The range of concentrations $\zeta$ over which the relaxation time data are provided for the three respective soft-colloid systems-I, II, and III, are distinct from each other. Relaxation behavior for each of the three materials is shown in figure 1 with respect to relative concentration. The three curves by construction meet at the same point on the y axis at $x=1$. It should also be noted that the relaxation curves in the Angell plot do not exactly meet at one point in the low-density side, though it might visually appear to be so in figure 1 . On a linear scale, it appears to be at $x=0$, though in reality these represent very small (nonzero) values of the packing fraction. The set of points representing $\tau_{\alpha}(x)$ vs $x=\zeta / \zeta_{\mathrm{g}}$ for the respective soft colloids are fitted the Vogel-Fulcher formula in the form (2.3). Each of the relaxation curves corresponding to the three materials I, II, and III is characterized by a corresponding set of values

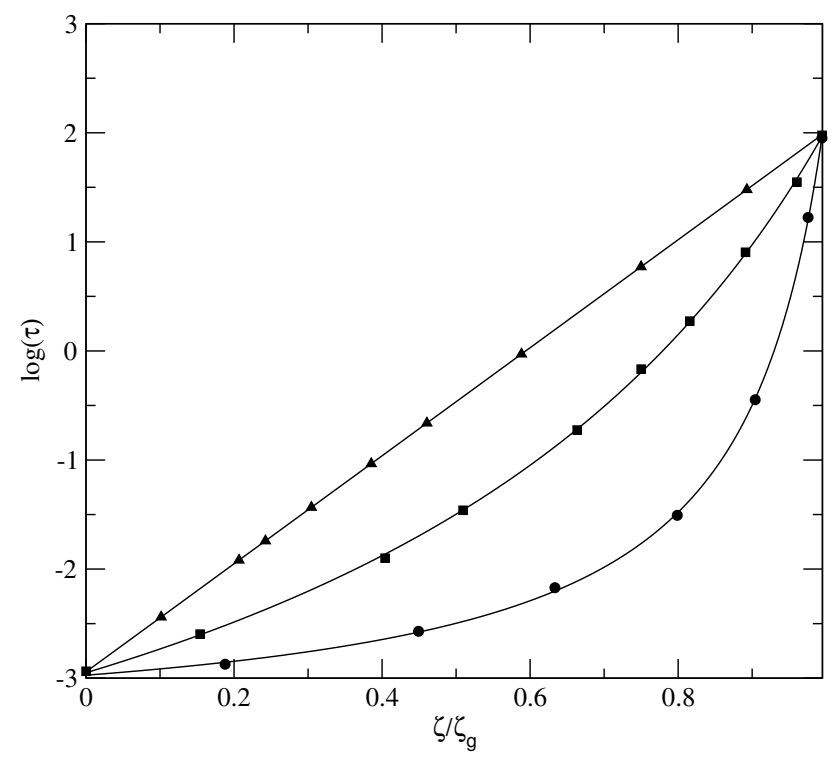

Figure 1. The Angell plot with the relaxation data for three soft colloid materials I, II, and III from [13], shown with filled circles, squares, and triangles, respectively. Fits with the form 2.1 in text for $\mathcal{B}=5$ and different choice for $\kappa=\zeta_{\mathrm{K}} / \zeta_{\mathrm{g}}$ are shown with solid lines. Corresponding $\kappa$ values are listed in table 2 . column 6 . 


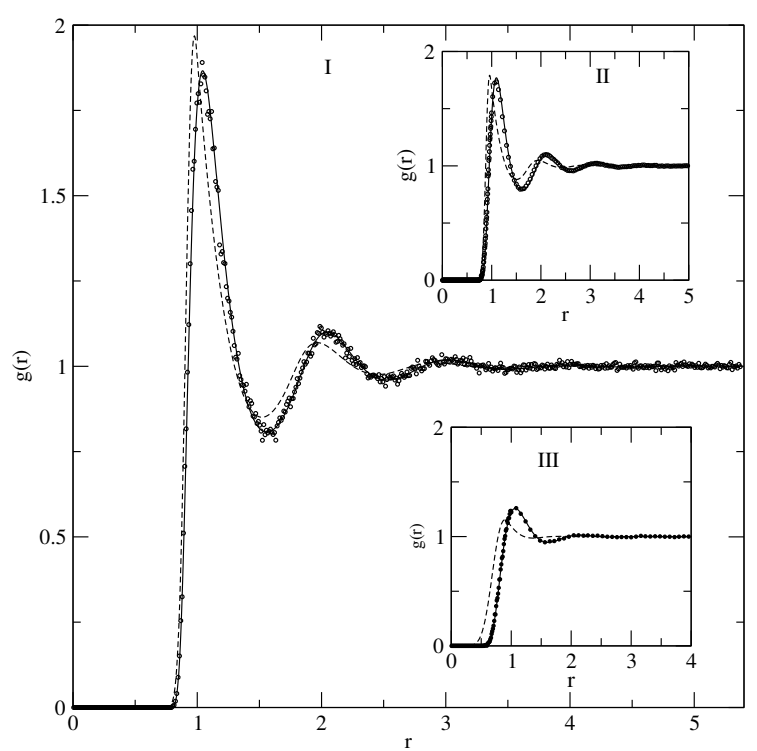

Figure 2. Pair correlation function $g(r)$ vs $r / \sigma$, for the three soft colloids I, II, and III (main panel and the two insets) interacting through a) soft sphere (solid) potential defined in equation (3.14) and b) Hertzian interaction (dashed) potential defined in equation 3.12. Parameters for the respective interaction potentials are given in table 4 and table 5 for the three soft colloids. The circles in each figure represent $g(r / \sigma)$ calculated from molecular dynamics simulation of soft spheres with interaction parameters $\left\{n, \epsilon^{*}\right\}$ as $\{12.1,0.571\},\{5.5,2.340\}$ and $\{3.1,1.440\}$, respectively, for soft colloids I, II, and III. In all three cases the relative concentration is $\zeta / \zeta^{*}=0.837$.

for $\{A, \kappa\}$ with the value for parameter $\mathcal{B}$ kept fixed at 5 . We find that these two parameters follow a linear fit $A=4.92 \kappa-4.88$. The corresponding fragility indices $m$ were obtained from the slope of these curves at the end point, i.e., $\zeta=\zeta_{\mathrm{g}}$. The ratio $\kappa=\zeta_{\mathrm{K}} / \zeta_{\mathrm{g}}$ for each curve and the corresponding fragility for the three respective soft colloids obtained this way are listed in table 2 The present scheme of determining fragility of the soft colloids only works for those materials corresponding to which the relaxations curves follow the form seen in an Angell plot, with a chosen value of the parameter $\mathcal{B}$ (which defines the so-called glass transition point $\zeta_{\mathrm{g}}$ ). Thus, if the relaxation does not follow this form for certain materials, such definitions for fragility do not work. To compare the relative trends in these results with respect to standard molecular glasses, in table 3 we show the fragility $m$ and the ratio $T_{\mathrm{g}} / T_{0}$ for three standard glass-forming materials, Salol, Glycerol and Silica [3] respectively. Here $T_{0}$ is the temperature

Table 1. Relaxation time data $\tau$ from figure $2 \mathrm{~b}$ of [13] in log scale vs the scaled concentration $\bar{\zeta}=\zeta / \zeta_{\mathrm{g}}$ (see text) for the three soft colloids SC-I, SC-II, and SC-III.

\begin{tabular}{|c|c|c|c|c|c|}
\hline \multicolumn{2}{|c|}{ SC- I } & \multicolumn{2}{c|}{ SC- II } & \multicolumn{2}{c|}{ SC- III } \\
\hline$\zeta$ & $\tau$ & $\zeta$ & $\tau$ & $\zeta$ & $\tau$ \\
\hline 0.016 & -2.972 & 0.016 & -2.950 & 0.016 & -2.892 \\
\hline 0.188 & -2.872 & 0.154 & -2.596 & 0.101 & -2.462 \\
\hline 0.449 & -2.570 & 0.404 & -1.899 & 0.207 & -1.952 \\
\hline 0.633 & -2.170 & 0.509 & -1.461 & 0.304 & -1.406 \\
\hline 0.798 & -1.507 & 0.816 & 0.273 & 0.460 & -0.610 \\
\hline 0.904 & -0.447 & 0.890 & 0.904 & 0.588 & -0.029 \\
\hline 0.975 & 1.223 & 0.960 & 1.547 & 0.892 & 1.442 \\
\hline 1.000 & 2.000 & 1.000 & 2.000 & 1.000 & 2.000 \\
\hline
\end{tabular}


Table 2. The ratio $\kappa=\zeta_{\mathrm{K}} / \zeta_{\mathrm{g}}$, obtained from the relaxation curves and the corresponding fragility $m$ for the three respective soft-colloids I, II, and III studied in [13].

\begin{tabular}{|c|c|c|}
\hline Soft-Colloid & The ratio $\kappa=\zeta_{\mathrm{K}} / \zeta_{\mathrm{g}}$ & Fragility $m$ \\
\hline I & 1.11 & 52.0 \\
\hline II & 1.70 & 12.1 \\
\hline III & 18.76 & 5.3 \\
\hline
\end{tabular}

Table 3. The ratio of the characteristic temperatures $T_{\mathrm{g}}$ and $T_{\mathrm{K}}$, fragility $m$ of the three standard molecular glass forming materials, are taken from [3]. The trend is similar to that between the ratio $\kappa$ and fragility $m$ as shown in the last two columns of table 2 for the soft colloids.

\begin{tabular}{|c|c|c|}
\hline Molecular Glass & The ratio $T_{\mathrm{g}} / T_{\mathrm{K}}$ & Fragility $m$ \\
\hline Salol & 1.13 & 66 \\
\hline Glycerol & 1.34 & 48 \\
\hline Silica & 3.26 & 25 \\
\hline
\end{tabular}

in the Vogel-Fulcher formula $\tau_{\alpha}=\tau_{0} \exp \left[A /\left(T-T_{0}\right)\right]$ signifying the divergence of relaxation time and it is determined for the three materials listed above from the relaxation data presented in [36]. The temperature $T_{0}$ is considered similar to the Kauzmann point $T_{\mathrm{K}}$ for a glass forming material. Comparing the results listed in table 2 with that of table 3 we note that with respect to fragility $m$, variation of $\zeta_{\mathrm{K}} / \zeta_{\mathrm{g}}$ in a colloidal glass is similar to that for $T_{\mathrm{g}} / T_{\mathrm{K}}$ in molecular glass formers. Hence, the trends seen in standard glass forming materials is the same as that for soft colloids.

\section{High-frequency elastic constants}

In this section, we consider the theoretical models for high-frequency or short time elastic constants for the soft colloids and the computation of these properties in terms of the pair correlation function. As a test, the input pair correlation function $g(r)$ is obtained in the present work with the choice of two different interaction potentials for the soft colloids.

\subsection{The model}

The Mountain-Zwanzig [15, 41, 42] theory calculates these quantities in terms of the interaction potential. Instantaneous response to stress in the disordered state is related to the corresponding frequency dependent viscosities [43]. Thus, the shear and bulk modulus are obtained from the generalized viscosities in the high-frequency limit and is denoted as $G_{\infty}(\zeta)$ and $K_{\infty}(\zeta)$ in what follows. The high-frequency limits of the respective elastic constants are obtained as

$$
\begin{aligned}
& G_{\infty}=\mathrm{i} \lim _{\omega \rightarrow \infty}\{\omega \eta(\omega)\}, \\
& K_{\infty}=K_{0}+\mathrm{i} \lim _{\omega \rightarrow \infty}\left\{\omega \eta_{V}(\omega)\right\},
\end{aligned}
$$

where $\eta(\omega)$ and $\eta_{V}(\omega)$ are respectively the frequency-dependent shear and bulk viscosities for the fluid. $K_{0}$ is the zero frequency bulk modulus obtained in terms of the adiabatic derivative

$$
K_{0}=-V\left(\frac{\partial P}{\partial V}\right)_{s} .
$$

For pairwise additive form of the interaction potentials, the total potential energy $U$ is expressed as a sum of two body terms and the averages on the right hand side of equations (3.1) and (3.2) are expressed in 
terms of pair correlation function $g(r)$ [44].

$$
\begin{aligned}
& G_{\infty}=\rho_{0} k_{\mathrm{B}} T+\frac{2 \pi}{15} \rho_{0}^{2} \int_{0}^{\infty} \mathrm{d} r g(r) \frac{\mathrm{d}}{\mathrm{d} r}\left[r^{4} \frac{\mathrm{d} u}{\mathrm{~d} r}\right], \\
& K_{\infty}=\frac{2}{3} \rho_{0} k_{\mathrm{B}} T+P+\frac{2 \pi}{9} \rho_{0}^{2} \int_{0}^{\infty} \mathrm{d} r g(r) r^{3} \frac{\mathrm{d}}{\mathrm{d} r}\left[r \frac{\mathrm{d} u}{\mathrm{~d} r}\right],
\end{aligned}
$$

where $\rho_{0}$ is the density and $P$ is the thermodynamic pressure for the simple model considered here and is identified with osmotic pressure for colloids. The latter is obtained with the standard expression [44]

$$
P=\rho_{0} k_{\mathrm{B}} T-\frac{2 \pi}{3} \rho_{0}^{2} \int_{0}^{\infty} \mathrm{d} r g(r) r^{3} \frac{\mathrm{d} u}{\mathrm{~d} r} .
$$

The Poisson's ratio has often been linked to relaxation behavior of a glass-forming system [36, 4547, 50, 51]. The Poisson ratio $v$ for the soft colloid material is defined in terms of the ratio of the elastic constants $\mu=K_{\infty} / G_{\infty}$ as,

$$
v=\frac{3 \mu-2}{2(3 \mu+1)} .
$$

In this paper, we consider the elastic response of the soft colloids over short times or equivalently, in the high-frequency limit. For this analysis, we use the data for three soft colloids in terms of the shear modulus $G_{\mathrm{p}}(\zeta)$ evaluated at frequency $\omega_{p}$. This frequency signifies the time scale of local particle motion. We identify the latter with short time or high-frequency response of the soft-colloid material and compare it with the corresponding high-frequency elastic constants obtained from the theoretical models based on microscopic statistical mechanics. The observed behaviours of the data of the elastic constant $G_{p}$ for the three respective soft-colloids are also reported [13] to be qualitatively similar to that of the high-frequency shear modulus $G_{\infty}$ for a set of glass forming (molecular) liquids. Hence, we use the simple model outlined above for the high-frequency elastic constant of a liquid in terms of the pair correlation function $g(r)$. We calculate both high-frequency shear modulus $G_{\infty}$ and bulk modulus $K_{\infty}$, respectively, for the soft colloids. The short-time elastic response of the liquid is described above with equations (3.4)- 3.7) in which the corresponding pair correlation functions are obtained using the microscopic two-body interaction potential as input. This also makes it possible to obtain the Poisson's ratio $v$ for the respective materials. Using the expressions on the right hand side of equations 3.4 -3.6 . we obtain the two elastic constants as follows:

$$
\begin{aligned}
G_{\infty} & =1+\mathcal{I}_{1}+\mathcal{I}_{2}, \\
\frac{3}{5} K_{\infty} & =1+\mathcal{I}_{1}-\mathcal{I}_{2},
\end{aligned}
$$

where the integrals $\mathcal{I}_{1}$ and $\mathcal{I}_{2}$ are obtained as functionals of the pair correlation function $g(s)$ and the dimensionless form of the interaction potential $\beta u(r) \equiv \bar{u}(s)$ as follows:

$$
\begin{aligned}
& \mathcal{I}_{1}[g, \bar{u}]=\frac{4}{5} \zeta \int_{0}^{\infty} \mathrm{d} s g(s) s^{3} \frac{\mathrm{d}}{\mathrm{d} s}\left[s \frac{\mathrm{d} \bar{u}(s)}{\mathrm{d} s}\right], \\
& \mathcal{I}_{2}[g, \bar{u}]=\frac{12}{5} \zeta \int_{0}^{\infty} \mathrm{d} s g(s) s^{3} \frac{\mathrm{d} \bar{u}}{\mathrm{~d} s} .
\end{aligned}
$$

In the above definitions, the radial distribution function $g(s)$ is expressed in terms of radial distance $r$ being scaled with a characteristic scale of length $\sigma$ for the chosen interaction potential. The energy $u$ is scaled with $k_{\mathrm{B}} T=\beta^{-1}$. Note that the data reported in [13] are only useful for comparison of the shear 
modulus $G_{\infty}(\zeta)$. As indicated above we consider here two possible bare interaction potentials to model the soft colloid materials. The characteristic parameters for the corresponding interaction potential are adjusted to fit the shear modulus data. The two interaction potentials and calculation of the corresponding pair correlation functions in the respective cases are briefly sketched below.

\subsection{The interaction potentials}

The two-body interaction potentials respectively used to model the soft colloids are as follows. 1. The Hertzian potential: Interaction between the colloid particles is given by

$$
u(r)=\epsilon_{0}\left(1-\frac{r}{r_{\mathrm{Hz}}}\right)^{5 / 2},
$$

in terms of two positive parameters $\left\{\epsilon_{0}, r_{\mathrm{Hz}}\right\}$. The length $r_{\mathrm{Hz}}$ represents a characteristic length scale at which the interaction potential $u(r)$ becomes zero and is used as an adjustable parameter for the potential. The potential energy $u(r)$ is expressed in units of $k_{\mathrm{B}} T$ as $\bar{u}(s)$ in terms of the scaled variable $s=r / \sigma$, where $\sigma$ is the length scale with which we define the concentration variable as $\zeta=\pi \sigma^{3} / 6$.

$$
\bar{u}(s)=\epsilon_{0}^{*}\left(1-\frac{s}{s_{\mathrm{Hz}}}\right)^{5 / 2},
$$

where $s_{\mathrm{Hz}}=r_{\mathrm{Hz}} / \sigma$. The energy scale $\epsilon_{0}$ in units of $k_{\mathrm{B}} T$, is obtained as $\epsilon_{0}^{*}=\epsilon_{0} /\left(k_{\mathrm{B}} T\right)$.

2. The soft sphere interaction. The potential [52, 53] is given by,

$$
u(r)=\epsilon_{0}\left(\frac{\sigma}{r}\right)^{n}
$$

in terms of two positive parameters $\left\{\epsilon_{0}, n\right\} . \sigma$ represents a characteristic microscopic length for the interaction potential. The soft sphere potential defined in equation 3.14 is written in units of $k_{\mathrm{B}} T$ using the scaled variable $s=r / \sigma$ as,

$$
\bar{u}(s)=\frac{\epsilon_{0}^{*}}{s^{n}},
$$

where $\epsilon_{0}^{*}=\epsilon_{0} /\left(k_{\mathrm{B}} T\right)$. In the $n \rightarrow \infty$ limit, the soft sphere potential reduces to the hard-sphere limit with the hard-sphere diameter $d \equiv \sigma$, and the concentration variable $\zeta=\pi \rho \sigma^{3} / 6$ becomes identical to the packing fraction $\phi$. For finite $n$, we have soft spheres which are identified with the deformable colloids.

The well-known Bridge function method is used to compute the pair correlation $g(s)$ for each of the above two types of interaction potentials. These are briefly described in Appendix A. In the soft sphere potential case, the pair function is calculated using two equivalent approaches. First, we show the theoretical results obtained from the bridge-function method of Rodger and Young [54]. Second, the static correlations are obtained through a direct computer simulation of the corresponding fluid. As an example, in the main panel of figure 2 we show the respective $g(r)$ vs $r / \sigma$ for all the three materials studied at $\zeta / \zeta^{*}=0.837$. The main panel shows the results for soft-colloid material I, while the two insets show the same respectively for soft colloids II and III. In each case of the main panel and the insets of figure 2, we show the $g(r)$ both for the Hertzian potential and for the soft spheres at the same value of $\zeta / \zeta^{*}$. The plot of $\bar{u}(s)$ vs $s$ for the soft sphere potentials corresponding to the three materials I, II, and III is displayed in the main panel figure 3 . The same results for the Hertzian potential are shown in the inset of figure 3 In table 5, for each of the respective soft colloid materials I, II, and III, we compare the lengths $s_{\mathrm{Hz}}$ for Hertzian potential and the sizes $\left(R_{0}\right)$ of the corresponding spheres[13].

\section{Fragility vs elasticity}

The relaxation and elastic properties for soft colloids are analyzed to determine their correlations as supported from experimental data. The high-frequency shear moduli $G_{\infty}$ for three soft colloid materials 


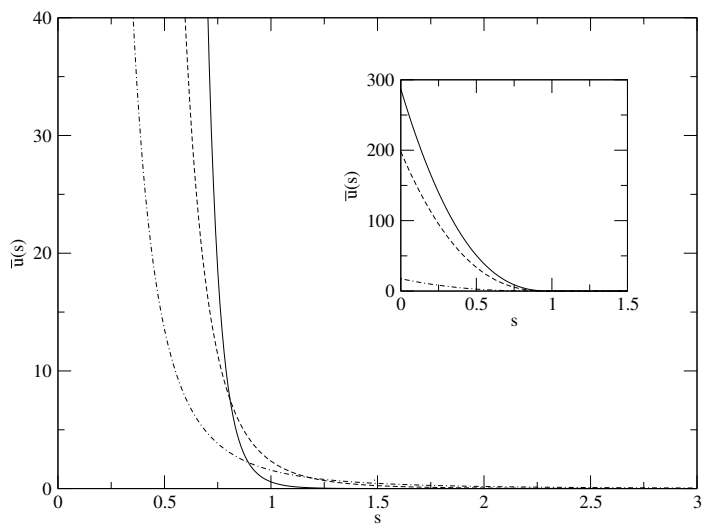

Figure 3. The soft sphere potential $\bar{u}(s)$ vs $s=r / \sigma$ with the characteristic parameters for interaction listed in table 4 for soft colloids I (solid), II (dashed), and III (dot-dashed), respectively. The inset shows the plot of the corresponding $\bar{u}(s)$ vs $s$ for the Hertzian potential having characteristic parameters listed in table 5

Table 4. The list of parameters for the soft-sphere potential defined in equation (3.14) needed for fitting the data of three soft colloids I, II, and III. The parameter $\zeta^{*}$ (column 3 ) is obtained through fitting the elasticity data as shown in figure 4-figure 6 (see text). The parameters for the corresponding soft sphere potential: energy $\epsilon_{0}^{*}=\epsilon_{0} / k_{\mathrm{B}} T$ (column 4$)$; softness index $n$ (column 5); $\kappa$ (column 6) is the ratio of the packing at the glass transition point $\left(\zeta_{\mathrm{g}}\right)$ to $\zeta^{*}$ (see text); $m$ (column 7$)$ is the fragility index.

\begin{tabular}{|c|c|c|c|c|c|c|}
\hline $\begin{array}{c}\text { Soft Colloid } \\
\text { material }\end{array}$ & $\begin{array}{c}\text { Data Range of } \\
\text { Concentration } \zeta\end{array}$ & $\begin{array}{c}\text { Parameter } \\
\zeta^{*}\end{array}$ & $\begin{array}{c}\text { energy } \\
\epsilon^{*}\end{array}$ & $\begin{array}{c}\text { softness } \\
n\end{array}$ & $\begin{array}{c}\zeta_{\mathrm{g}} / \zeta^{*}= \\
\kappa\end{array}$ & $\begin{array}{c}\text { Fragility } \\
m\end{array}$ \\
\hline I & $0.300-0.540$ & 0.500 & 0.571 & 12.1 & 1.11 & 52.0 \\
\hline II & $0.264-0.400$ & 0.100 & 2.340 & 5.5 & 6.65 & 12.1 \\
\hline III & $0.270-0.430$ & 0.054 & 1.440 & 3.1 & 12.46 & 5.3 \\
\hline
\end{tabular}

Table 5. The list of parameters for Hertzian potential defined in equations (3.12) -3.13 needed for fitting the data of three soft colloids I, II, and III. $\zeta^{*}$ (column 3 ) is obtained through fitting the elasticity data as shown in figure 46 (see text). The parameters for corresponding Hertzian potential : energy $\epsilon_{0}^{*}=\epsilon_{0} / k_{\mathrm{B}} T$ (column 4); $s_{\mathrm{Hz}}$ (column 5) is the length at which the potential $\bar{u}(s)$ is zero. The length $R_{0}$ (column 6) denotes the radius of the deformable spheres for the three respective soft colloids as reported in [13]. The lengths $s_{\mathrm{Hz}}$, and $R_{0}$, are scaled so that for material I, these quantities are unity by choice. Finally, $m$ (column 7) is the fragility of the soft colloids shown here for comparison.

\begin{tabular}{|c|c|c|c|c|c|c|}
\hline $\begin{array}{c}\text { Soft Colloid } \\
\text { studied }\end{array}$ & $\begin{array}{c}\text { Data Range of } \\
\text { Concentration } \zeta\end{array}$ & $\begin{array}{c}\text { Parameter } \\
\zeta^{*}\end{array}$ & $\begin{array}{c}\text { energy } \\
\epsilon_{0}^{*}\end{array}$ & $\begin{array}{c}\text { range } \\
s_{\mathrm{Hz}}\end{array}$ & $\begin{array}{c}\text { Radius } \\
R_{0}(\mathrm{~A})\end{array}$ & $\begin{array}{c}\text { Fragility } \\
m\end{array}$ \\
\hline I & $0.300-0.540$ & 0.50 & 287 & 1.00 & 1.00 & 52.0 \\
\hline II & $0.264-0.400$ & 0.10 & 198 & 0.98 & 0.97 & 12.1 \\
\hline III & $0.270-0.430$ & 0.054 & 17.6 & 0.96 & 0.84 & 5.3 \\
\hline
\end{tabular}

are fitted with the formulae presented in equations $(3.8)-(3.11)$. The pair correlation function $g(s)$ is the key input in this and is determined by the interaction potential between the colloidal particles. Note that for the soft sphere potential in the form, $\bar{u}(s) \sim s^{-n}$, the two integrals $\mathcal{I}_{1}$ and $\mathcal{I}_{2}$, respectively obtained in (3.10) and 3.11), are related simply as $\mathcal{I}_{1}=-(n / 3) \mathcal{I}_{2}$. In this case, the elastic constants $G_{\infty}(\zeta)$ and $K_{\infty}(\zeta)$ given in equations 3.4 - 3.5 respectively reduce to the forms $G_{\infty}=1+\Delta(n-3) / 5$, and $K_{\infty}=5 / 3+\Delta(n+3) / 3$, in terms of $\Delta=P-1$. For $n \geqslant 3, G_{\infty}$ decreases with an increase of $P$, and is requred for stability. 


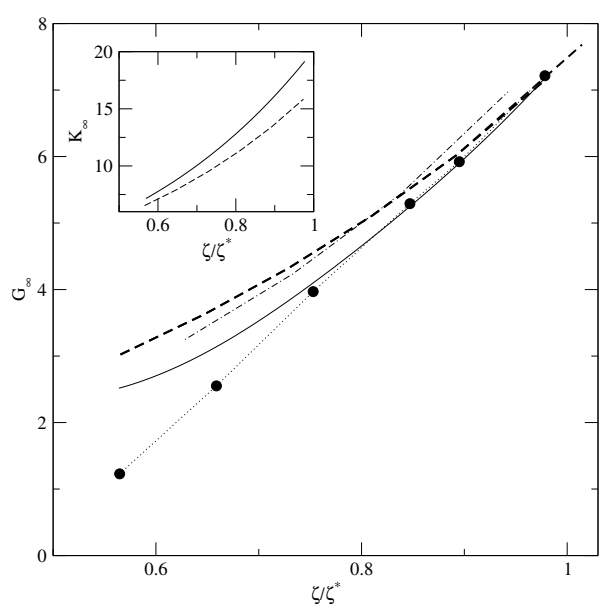

Figure 4. Main panel: Theoretical results for shear modulus $G_{\infty}(\zeta)$ in units of $\rho_{0} k_{\mathrm{B}} T$ vs scaled concentration $\zeta / \zeta^{*}$ (see text). We use equation 3.8 with structural inputs of $g(r)$ obtained from (i) the bridge function method for soft sphere interactions(solid line); (ii) molecular dynamics simulations with soft sphere interactions (dot-dashed); and (iii) the bridge function method for Hertzian potential (dashed). Comparison with shear modulus data for $G_{\mathrm{p}}(\zeta)$ for soft colloids I taken from figure $3 \mathrm{~b}$ of [13]; data scaled with a material-dependent factor for each system: filled circles are connected with the dotted line. Insets: theoretical results from equation (3.9) for bulk modulus $K_{\infty}(\zeta)$ in units of $\rho_{0} k_{\mathrm{B}} T$ vs scaled concentration $\zeta / \zeta^{*}$, with the same input $g(r)$ as obtained from fitting the shear modulus data for the material shown in the corresponding main panel: for soft sphere potential (solid line) and Hertzian potential (dashed line) bridge function method.

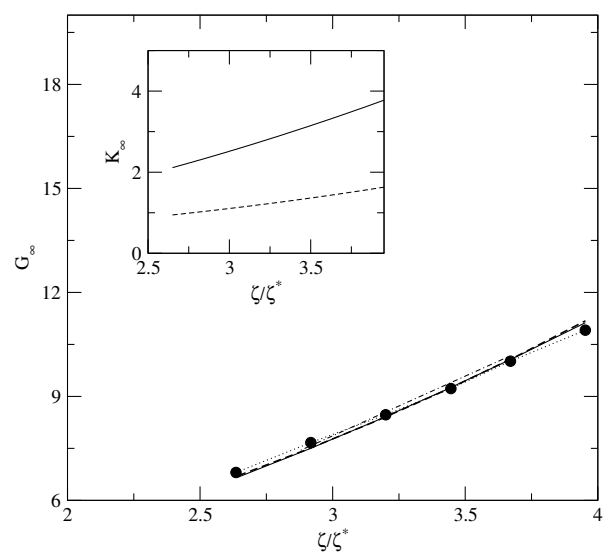

Figure 5. The same as figure 4 for soft colloid II of [13]

For the Hertzian potentials defined in equation (3.13), the shear modulus data are fitted by adjusting parameters, $\left\{s_{\mathrm{Hz}}, \epsilon_{0}^{*}\right\}$, while for the soft sphere potential defined in $(3.15)$ the corresponding parameters are $\left\{n, \epsilon_{0}^{*}\right\}$. The results are listed in tables 4 and 5 . For a particular soft colloid material (I, II, or III), the parameter values corresponding to a chosen interaction potential (respectively being Hertzian or soft-sphere) are kept fixed while fitting the shear modulus data for that material over the whole range of density values. We obtain the respective set of parameters for the interaction potential so that the corresponding pair functions $g(s)$ in the theoretical formulae produce the best fit with the $G_{\infty}$ data over the whole density range studied for a particular soft colloid material. With a suitable choice of the parameter $\zeta^{*}$ for each case, we match the $G_{\mathrm{p}}(\zeta)$ data for the three materials. Corresponding to the specific set of parameter values for the potential $\bar{u}(s)$, the pair functions $g(s)$ (at different densities) fit the shear moduli $G_{\mathrm{p}}(\zeta)$ data. In figure 4-figure 6, we show the comparison between experimental data for $G_{\infty}$ of the three respective soft colloids with the corresponding theoretical results obtained using a) the 


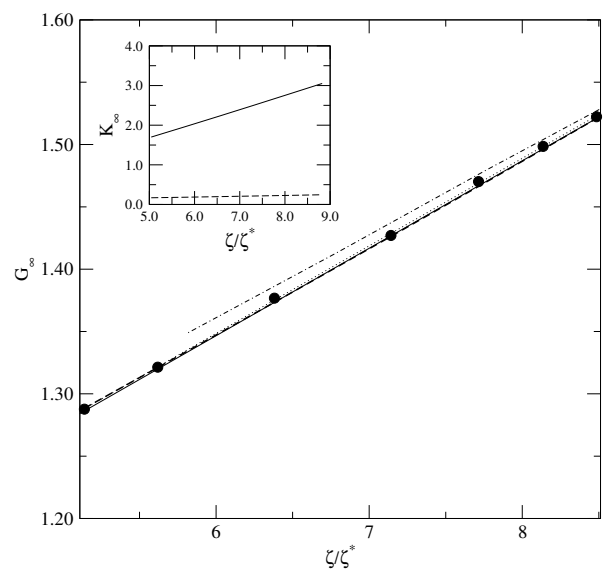

Figure 6. The same as figure 4 for soft colloid III of [13]

Hertzian and b) the soft sphere interaction potentials. Next, the same $g(r)$ in each respective case is used to obtain the corresponding bulk modulus $K_{\infty}(\zeta)$. This procedure is followed for each of the three Soft colloids, using the formulae (3.5), 3.9), and 3.10)-(3.11). The bulk moduli $K_{\infty}$ obtained for the three samples SC-I, II, and III are shown respectively in the insets of figure 4-figure 6 For all three materials I, II, and III, the bulk modulus obtained for the Hertzian potential case is lower than the corresponding results for the soft sphere potential. In figure 7 we show how the ratio $\mu=K_{\infty}(\zeta) / G_{\infty}(\zeta)$ changes with concentration $\zeta$ for the three soft materials in I, II, and III. Since the ratio $\mu$ is material dependent, it is not very sensitive to an increase of the concentration $\zeta$.

The optimum parameter values for the respective interaction potentials, obtained by matching shear modulus data with the theoretical formula (3.4), signify a particle size for the respective materials and correlate corresponding radii $R_{0}$. The cutoff length $s_{\mathrm{Hz}}$ of the Hertzian potential, and $R_{0}$, are shown in table 5. The results shown are scaled so that for material I, these quantities are unity. The respective

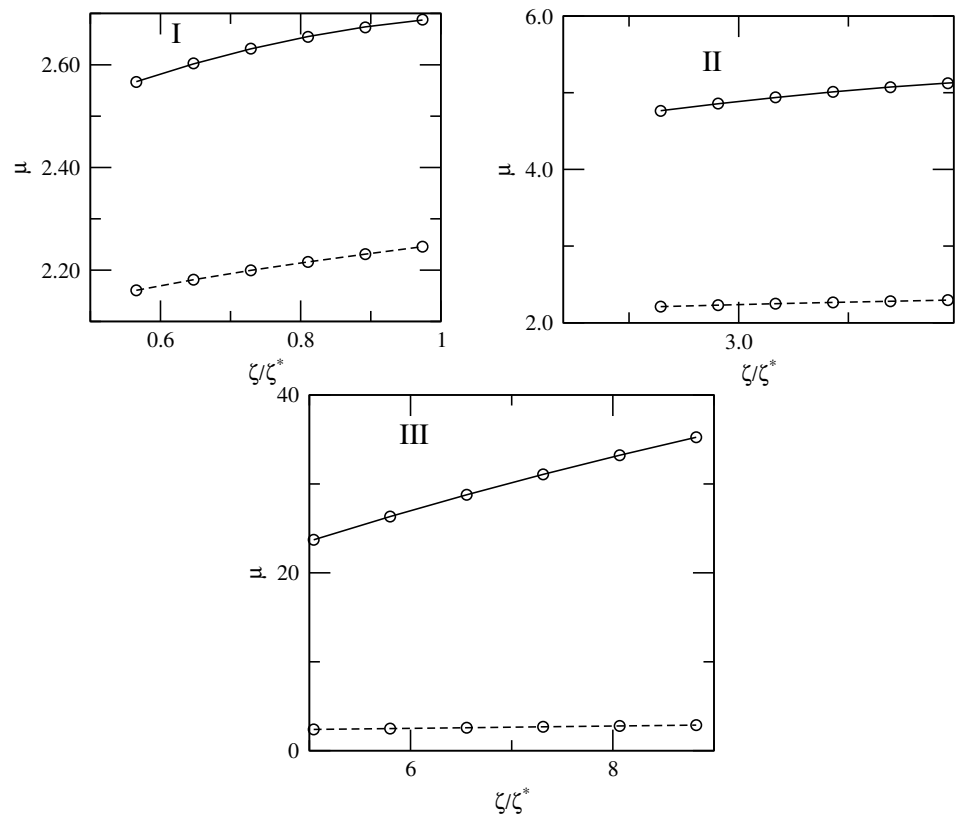

Figure 7. The ratio $\mu=K_{\infty}(\zeta) / G_{\infty}(\zeta)$ vs $\zeta / \zeta^{*}$ for the three materials : I, II, and III. The results correspond to the calculation done with $g(r)$ for soft sphere potential (solid) and Hertzian potential (dashed) in each figure. 


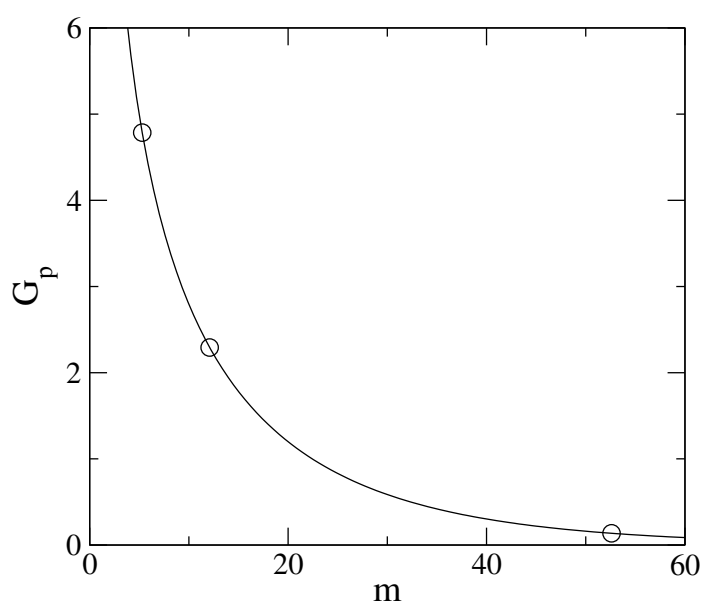

Figure 8. The plot of the shear modulus $G_{p}$ vs fragility $m$ for the three soft colloids I, II, and III (see text). Data taken from figure $3 \mathrm{a}$ of [13] and scaled with a factor of $\tilde{\sigma}^{3} /\left(k_{\mathrm{B}} T\right)$. Here $\tilde{\sigma}=0.1 R_{0}$ (see text).

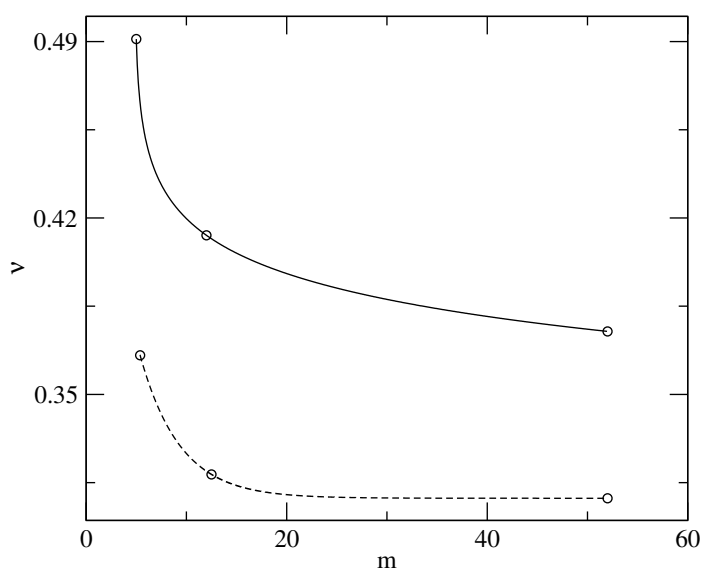

Figure 9. The plot of the Poisson's ratio $v$ vs the fragility $m$ for the three materials studied in the present work, for the soft sphere potential (solid) and Hertzian potential (dashed).

variations of $s_{\mathrm{Hz}}$ and $R_{0}$ with the fragility $m$ of the three soft colloids I, II, and III show the same qualitative trend. Similarly, the softer is the potential, the lower is the value of $m$. With the soft sphere potential, for increasing values of softness index $n$, the fragility $m$ increases. This also agrees with the view that hard-sphere systems $(n \rightarrow \infty)$ are most fragile. In figure 8 we present how the shear modulus for the respective three soft colloids I, II, and III correlates with the corresponding fragility values. This figure presents a plot of $G_{\infty}$ (expressed in units $k_{\mathrm{B}} T / \tilde{\sigma}^{3}$ ) at $\zeta=\zeta_{\mathrm{g}}$ vs the fragility $m$ for the corresponding material. The experimental shear modulus data displayed here are taken from figure 3a of [13] and these form the common basis for finding the optimum parameter values for both types of potentials. The trend of correlation of $m$ with $G_{\infty}$ is shown here is similar to that of the Poisson's ratio which we discuss next. We calculate the Poisson's ratio $v$ using the formula equation (3.7) at the glass transition point $\zeta=\zeta_{\mathrm{g}}$. In figure 9 a plot of $v$ at the glass transition point $\zeta_{\mathrm{g}}$ with respect to the corresponding value of the fragility index $m$ is shown for both types of interaction potentials.

In all cases, corresponding to both types of interaction potentials, soft sphere repulsive and Hertzian, the Bulk modulus is larger than the corresponding shear modulus. However, for the soft sphere interaction case, the ratio is much larger and hence the Poisson ratio remains close to $0.4 \sim 0.5$. For the Hertzian potential, the difference between bulk and shear moduli is less pronounced and hence the variation in Poisson's ratio is wider. The qualitative trends for correlations of either $G_{\infty}$ or $v$ with the fragility is observed to be same. For the sake of comparison, figure 10 shows the correlation between the same 


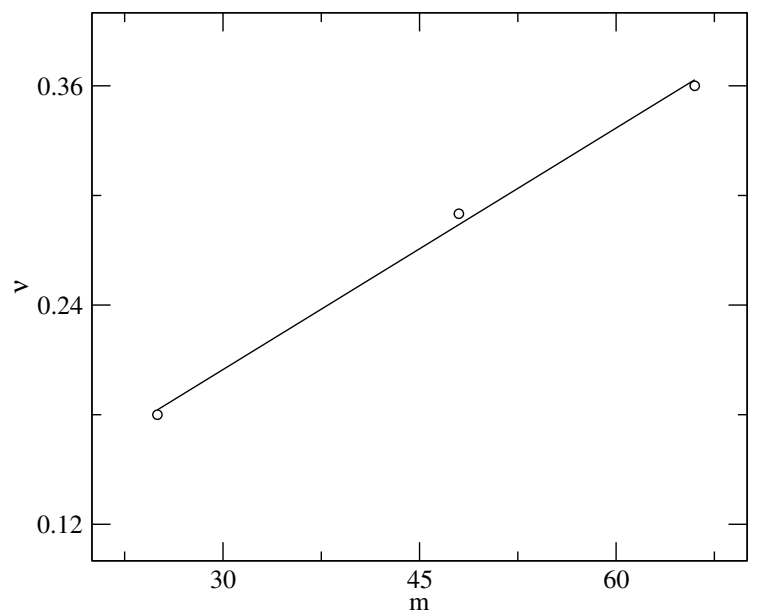

Figure 10. The plot of the Poisson's ratio $v$ vs the fragility $m$ for the three molecular glass formers. In the increasing order of fragility (m) these are Silica, Glycerol, and Salol taken from [55]. The results in each case are shown as points and the lines are a guide to the eye. The trends of the data are opposite for the molecular systems and soft colloids.

quantities, $v$ and $m$ in molecular systems. Here, the results for three standard molecular glass-forming systems [13] as taken from the literature [36, 37, 55] are displayed. In soft colloids, Poisson's ratio $v$ varies with respect to $m$ in a manner which is opposite to what is observed in molecular glass-forming systems. These opposing trends are discussed further in the next section.

\section{Discussion}

The static and dynamic behaviours of colloidal hard-sphere systems [56-58] have similarities with those seen in molecular liquids. Experimental studies of Mattsson et al. [13] demonstrated that even deformable colloidal suspensions exhibit the dynamical behaviour qualitatively similar to a fluid of nondeformable spheres. Relaxation times grow with concentration $(\zeta)$ of constituent particles in a soft colloid material and the concept of fragility is directly extended to suspensions of deformable colloidal particles in terms of density. We identify each of the relaxation curves displayed in the so-called "Angell-plot" with a corresponding set of parameters for a (chosen) type of two-body interaction-potential. We do this by fitting the short time (high-frequency) shear modulus data [13] for the respective materials using theoretical formulae involving pair correlation function $g(r)$ for the system. The appropriate $g(r)$ for each soft-colloid is chosen by adjusting the parameters for interaction potential. We have chosen in our study the Hertzian potential as well as soft sphere potential. For the same pair potential, the bulk moduli $K_{\infty}(\zeta)$ are calculated in each case and hence the Poisson's ratio is calculated.

It will be useful to pinpoint the key approximation involved in the simple model for the soft colloids as we presented here. The system of deformable soft spheres is modelled here in terms of a system of microscopic particles interacting through a bare two-body potential. For the latter, we have made the simplest possible choices, namely soft sphere and Hertzian types. Thus, the deformable property of the spheres is mapped in terms of the softness of respective interaction potentials, characterized by the corresponding strength and range for each system. The elastic constants or pressure, respectively given in equations (3.4)-(3.5), and (3.6) are expressed in terms of the two point pair correlation function $g(r)$ for the corresponding interaction potential. With the appropriate choice of interaction parameters in the respective cases, obtained by fitting the shear modulus data, the number density or concentration $\zeta$ is the key variable in the theoretical model. In the present work we approximate the pair correlation function $g(r)$ with a simple form for an isotropic system with uniform density. Hence, $g(r)$ depends on the parameters of interaction potential and the average number density. A system of deformable spheres in reality may have a nonuniform state and the pair correlation function $g(\mathbf{r})$ will have to account for the concentration 
dependent change and local packing in a self-consistent manner. This will be a way of accounting for the variations in the size of particles due to the local pressure. A self-consistent formulation of pair function in terms of local density, going beyond the simple isotropic form $g(r)$ used here for uniform density state, will be needed to make a model for the long-time dynamics of the soft colloid materials. In the present context, we use the simplest model for the short-time behaviour of the soft colloid materials.

The correlation observed here linking the fragility $m$ and softness index $n$ or the Poisson's ratio $v$ in the glass forming system, is based on the analysis of experimental data as well as on the use of simple models. The theoretical model which links the interaction potential to elastic constants in this work, was originally proposed by Zwanzig and Mountain to explain the short time elastic response of the liquid in terms of the interaction potential. Deductions of these formulae for the elastic constants do not require explicit models for the dynamics for the soft colloids since the theory primarily focuses on the short time or high-frequency elastic response of the liquid. Time correlation functions in the Green-Kubo expressions for the viscosity are only calculated here in the infinite frequency or zero time limit and hence one is dealing with only static correlations. For the time-dependent behaviour or finite frequency response of soft materials valid over different time scales, modelling of the dynamics [59] would be needed. For the long time elastic response in a frozen state, mode coupling contributions [60] coming from nonlinear coupling of density fluctuations [61] are important. For the Brownian dynamics of the soft colloid particles, the relaxation times observed here are long and it indicates that strongly cooperative motions occur. The glassy dynamics in soft colloids was studied using a microscopic model, such as the mode coupling theory (MCT) [62, 63]. In analogy with these models, we have chosen soft repulsive spheres in our theoretical analysis while comparing with experimental data.

For models in which interaction between colloid particles is chosen to be soft sphere potential, the relaxation data indicate the lowering of the fragility index $m$ with decreasing values of softness index $n$. A similar dependence of the softness of interaction potential on the fragility of an atomic system was obtained using numerical studies of binary Lennard-Jones systems [20]. This trend, however, is not universal. In some simulations of binary mixtures [64], the fragility was even reported to be independent of softness of the potential. Temperature dependence of the diffusion coefficients for various $n$ collapses in this case onto a universal curve. Thus, as concerns the link between fragility and softness index, our findings do match with some, but not all, numerical results obtained in case of molecular systems. It is also worth to note here that the fragility for a molecular system is defined with the slope of the corresponding relaxation curve in the Angell-plot at $T=T_{\mathrm{g}}$. In the present work, following [13], fragility is calculated through density dependence, at $\zeta=\zeta_{\mathrm{g}}$. Correlation between the Poisson's ratio and fragility was found [32, 37, 45, 50, 65] to be non-universal even within only molecular systems. Thus, while there exist a fundamental link between fragility and elasticity through the basic interaction potential, and the underlying structure of the liquid influences its long time dynamics, and the observed correlation between $m$ and $v$ demonstrated in the present work is still at the level of a hypothesis.

From a theoretical perspective, the observed link between the fragility of a metastable liquid and its elastic coefficients is a manifestation of structural effects on the dynamics of a many-particle system. The elastic response of the fluid approaching the glass transition signifies a solid-like behavior. Fragility index, on the other hand, is related to the relaxation process in the liquid state near the so-called glass transition point. Developing a common basis for both elasticity and fragility involves understanding the process of a rigidity-transformation of the metastable liquid into an amorphous solid-like state [66]. The success of basic theoretical models in comprehending this transition is only partial so far.

\section{Acknowledgement}

AM acknowledges UGC-BSR fellowship, and LP acknowledges CSIR, India for financial support. SPD acknowledges support under JC Bose fellowship grant. 


\section{A. The pair-correlation function}

In this Appendix we very briefly describe the standard procedure followed for calculation of the pair correlation functions for the chosen interaction potentials.

\section{A.1. The Bridge functions for pair correlation}

We use here the bridge function method of standard liquid state theory to obtain $g(r)$ for a soft sphere system with the chosen values of the parameters $n$ and $\epsilon_{0}^{*}$. We also compute the static functions through direct molecular dynamics simulations for the chosen soft sphere potential. We briefly outline here the bridge function method as well as the simulation approach which we follow to compute the pair function $g(r)$. The total correlation function for the fluid is defined as, $h(r)=g(r)-1$. The direct correlation function denoted as $c(r)$ [44] is linked to $h(r)$ by Ornstein-Zernike $(\mathrm{O}-\mathrm{Z})$ relation

$$
h(r)=c(r)+\int h\left(r-r^{\prime}\right) c\left(r^{\prime}\right) \mathrm{d} r^{\prime}
$$

The set of equations for $c(r), h(r)$ are closed by choosing a closure relation through the introduction of a bridge function $B(r)$. The closure equation is chosen in the form

$$
y(r)=\mathrm{e}^{h(r)-c(r)+B(r)},
$$

where $y(r)=g(r) \exp \left[u(r) /\left(k_{\mathrm{B}} T\right)\right]$ and $B(r)$ is the bridge function introduced in defining the closure. To construct $B(r)$, various approximations have been devised in the literature. For soft sphere potentials, a successful approach is the Rodger-Young bridge function [54, 67]. We solve the O-Z equation (A.1) numerically with the Rodger and Young closure. The corresponding results for $g(r)$ obtained agree well with the same quantity calculated using computer simulation. Our results show that the elastic properties are very sensitive to the pair function.

In the computer simulations, we study a system of particles interacting via inverse power law potential given by equation (3.14). We set a cut off of the potential at $r_{\text {cut }}=2.5 \sigma$ and study the dynamics of one thousand particles. Using the molecular dynamics simulation, the equilibrated samples are generated for different state points. The potential parameters are adjusted to obtain the $g(r)$ that provides, through the formula (3.8), the best fit to the shear modulus data reported in [13]. The equilibrated samples are initially generated for different state points at $n=12.1, T^{*}=1.75 ; n=5.5, T^{*}=0.43$; and $n=3.112, T^{*}=0.639$ using Nosé-Hoover thermostat at constant NVT. By evolving further, using NVE integrator, for each state point at least 100 independent samples well separated by the structural relaxation times are obtained. The time step used throughout the simulation is 0.001 in Lennard-Jones time units. In all the three state points, we find that the systems do not crystallize as shown by the pair correlation function. The pair correlation function is calculated by averaging over independent samples.

\section{References}

1. Angell C.A., Science, 1995, 267, 1924-1935, doi $10.1126 /$ science.267.5206.1924

2. Turnbull D., Fisher J.C., J. Chem. Phys., 1949, 17, 71-74, doi $10.1063 / 1.1747055$

3. Böhmer R., Angell C.A., Phys. Rev. B, 1992, 45, 10091-10094, doi:10.1103/PhysRevB.45.10091

4. Böhmer R., Angell C.A., Phys. Rev. B, 1993, 48, 5857-5864, doi:/10.1103/PhysRevB.48.5857.

5. Nagel S.R., Rev. Mod. Phys., 2017, 89, 025002 (23 pages), doi $10.1103 /$ RevModPhys.89.025002

6. Gokhale S., Sood A.K., Ganapathy R., Adv. Phys., 2016, 65, 363-452, doi:10.1080/00018732.2016.1200832

7. Bonnecaze R.T., Cloitre M., Adv. Polymer Sci., 2010, 236, 117-161, doi:10.1007/12_2010_90

8. Gnan N., Zaccarelli E., Nat. Phys., 2019, 15, 683-688, doi 10.1038/s41567-019-0480-1.

9. Xu N., Haxton T.K., Liu A.J., Nagel S.R., Phys. Rev. Lett., 2009, 103, 245701 (4 pages), doi $10.1103 /$ PhysRevLett.103.245701

10. Schmiedeberg M., Haxton T.K., Nagel S.R., EPL, 2011, 96, 36010 (5 pages), doi $10.1209 / 0295-5075 / 96 / 36010$

11. Hunter G.L., Weeks E.R., Rep. Prog. Phys., 2012, 75, 066501 (30 pages), doi $10.1088 / 0034-4885 / 75 / 6 / 066501$ 
12. Lu P.J., Weitz D.A., Annu. Rev. Condens. Matter Phys., 2013, 4, 217-233, doi 10.1146/annurev-conmatphys-030212-184213.

13. Mattsson J., Wyss H.M., Fernandez-Nieves A., Miyazaki K., Hu Z., Reichman D.R., Weitz D.A., Nature, 2009, 462, 83-86, doi $10.1038 /$ nature08457

14. Shao Z., Negi A.S., Osuji C.O., Soft Matter, 2013, 9, 5492-5500, doi:10.1039/C3SM50209K

15. Zwanzig R., Mountain R.D., J. Chem. Phys., 1965, 43, 4464-4471, doi $10.1063 / 1.1696718$

16. Casalini R., J. Chem. Phys., 2012, 137, 204904 (4 pages), doi:10.1063/1.4768267

17. Casalini R., Mohanty U., Roland C.M., J. Chem. Phys. 2006, 125, 014505 (9 pages), doi 10.1063/1.2206582.

18. Casalini R., Roland C.M., Phys. Rev. E, 2005, 72, 031503 (4 pages), doi:10.1103/PhysRevE.72.031503

19. Wales D.J., Energy Landscapes, Cambridge University Press, Cambridge, New York, 2003.

20. Shi Z., Debenedetti P.G., Stillinger F.H., Ginart P., J. Chem. Phys., 2011, 135, 084513 (9 pages), doi $10.1063 / 1.3627148$.

21. Singh Y., Stoessel J.P., Wolynes P.G., Phys. Rev. Lett., 1985, 54, 1059-1062, doi $10.1103 /$ PhysRevLett.54.1059

22. Löwen H., J. Phys. C, 1990, 2, 8477-8484, doi $10.1088 / 0953-8984 / 2 / 42 / 024$

23. Kaur C., Das S.P., Phys. Rev. E, 2002, 65, 026123 (7 pages), doi: 10.1103/PhysRevE.65.026123

24. Martin P.C., Parodi O., Pershan P.S., Phys. Rev. A, 1972, 6, 2401-2420, doi/10.1103/PhysRevA.6.2401

25. Das S.P., Phys. Rev. E, 1999, 59, 3870-3875, doi 10.1103/PhysRevE.59.3870

26. Srivastava S., Das S.P., Phys. Lett. A, 2001, 286, 76-79, doi:10.1016/S0375-9601(01)00391-7.

27. Tarazona P., Mol. Phys., 1984, 52, 81-96, doi $10.1080 / 00268978400101071$

28. Kirkpatrick T.R., Wolynes P.G., Phys. Rev. A, 1987, 35, 3072-3080, doi 10.1103/PhysRevA.35.3072

29. Premkumar L., Bidhoodi N., Das S.P., J. Chem. Phys., 2016, 144, 124511 (8 pages), doi $10.1063 / 1.4944825$

30. Jae-Hyung J., Tejedor V., Burov S., Barkai E., Selhuber-Unkel C., Berg-Srensen K., Oddershede L., Metzler R. Phys. Rev. Lett., 2011, 106, 048103 (4 pages), doi:10.1103/PhysRevLett.106.048103

31. Priya M., Das S.P., Phys. Rev. E, 2007, 76, 061501 (8 pages), doi:10.1103/PhysRevE.76.061501

32. Novikov V.N., Sokolov A.P., Phys. Rev. B, 2006, 74, 063203, doi 10.1103/PhysRevB.74.064203

33. Scopigno T., Ruocco G., Sette F., Monaco G., Science, 2003, 302, 849-852, doi $10.1126 /$ science.1089446

34. Tobolsk A., Powell R.E., Eyring H., In: Frontiers in Chemistry, Vol. 1, Burk R.E., Grummit O. (Eds.), Interscience, 1943, 125-190.

35. Nemilov S.V., Thermodynamic and Kinetic Aspects of the Vitreous State, 1995, CRC Press, Boca Raton, doi $10.1201 / 9781351077286$

36. Novikov V.N., Sokolov A.P., Nature, 2004, 431, 961-963, doi:10.1038/nature02947

37. Novikov V.N., Ding Y., Sokolov A.P., Phys. Rev. E., 2005, 71, 061501 (12 pages), doi 10.1103/PhysRevE.71.061501.

38. Srivastava S., Das S.P., Phys. Lett. A, 2001, 286, 76-79, doi 10.1016/S0375-9601(01)00391-7.

39. Oldekop W., Glass Sci. Technol., 1957, 30, 8-14.

40. Laughlin W.T., Uhlmann D.R., J. Phys. Chem., 1972, 76, 2317-2325, doi:10.1021/j100660a023

41. Mountain R.D., Zwanzig R., J. Chem. Phys., 1966, 44, 2777-2779, doi 10.1063/1.1727124

42. Schofield P., Proc. Phys. Soc., 1966, 88, 149-170, doi 10.1088/0370-1328/88/1/318.

43. Boon J., Yip S., Molecular Hydrodynamics, Dover, New York, 1991.

44. Hansen J.P., McDonald I.R., Theory of Simple Liquids, Elsevier, Amsterdam, 2006.

45. Ostergaard M.B., Hansen S.R., Januchta K., To T., Rzoska S.J., Bockowski M., Bauchy M, Smedskjaer M.M., Materials, 2019, 12, 2439 (12 pages), doi $10.3390 / \mathrm{ma12152439}$

46. Sun Q., Hu L., Zhou C., Zheng H., Yue Y., J. Chem. Phys., 2015, 143, 164504, doi:10.1063/1.4934228.

47. Ngai K.L., Wang L., Liu R., Wang W.H., J. Mol. Liq., 2015, 205, 37-41, doi 10.1016/j.molliq.2014.08.038

48. Malfait W.J., Sanchez-Valle C., Chem. Geol., 2013, 346, 72-80, doi 10.1016/j.chemgeo.2012.04.034

49. Souri D., Salehizadeh S.A., J. Therm. Anal. Calorim., 2013, 112, 689-695, doi $10.1007 / \mathrm{s} 10973-012-2613-y$

50. Nemilov S.V., J. Non-Cryst. Solids, 2007, 353, 4613-4632, doi 10.1016/j.jnoncrysol.2007.08.045.

51. Yannopoulos S.N., Johari G.P., Nature, 2006, 442, E7-E8, doi:10.1038/nature04967

52. Glaser M.A., Grason G.M., Kamien R.D., Kosmrlj A., Santangelo C.D., Ziherl P., EPL, 2007, 7846004 (5 pages), doi:10.1209/0295-5075/78/46004

53. Kosmrlj A., Pauschenwein G.J., Kahl G., Ziherl P., J. Phys. Chem. B, 2011, 115, 7206-7217, doi $10.1021 /$ jp108806v

54. Rodgers F.J., Young D.A., Phys. Rev. A, 1984, 30, 999-1007, doi 10.1103/PhysRevA.30.999

55. Bóhmer R., Ngai K.L., Angell C.A., Plazek D.J., J. Chem. Phys., 1993, 99 4201-4209, doi 10.1063/1.466117

56. Pusey P.N., Liquids, Freezing and the Glass Transition, Levisque D., Hansen J.P., Zinn-Justin J. (Eds.), Elsevier, New York, 1991. 
57. Hansen J.P., Löwen H., Annu. Rev. Phys. Chem., 2000, 51, 209-242, doi:10.1146/annurev.physchem.51.1.209

58. Dijkstra M., Roji R.V., Evans R., Phys. Rev. E, 1999, 59, 5744-5771, doi 10.1103/PhysRevE.59.5744.

59. Wyss H.M., Miyazaki K., Mattsson J., Hu Z., Reichman D.R., Weitz D.A., Phys. Rev. Lett., 2007, 98, 238303 (4 pages), doi 10.1103/PhysRevLett.98.238303.

60. Das S.P., Statistical Physics of Liquids at Freezing and Beyond, Cambridge University Press, New York, 2011.

61. Dyre J.P., Wang W.H., J. Chem. Phys. 2012, 136, 224108 (5 pages), doi:10.1063/1.4724102

62. Chen K., Saltzman E.J., Schweizer K.S., Ann. Rev. Cond. Matt. Phys., 2010, 1, 277-300, doi 10.1146/annurev-conmatphys-070909-104110

63. Das S.P., Rev. Mod. Phys., 2004, 76, 785-851, doi 10.1103/RevModPhys.76.785

64. Michele C.D., Sciortino F., Coniglio A., J. Phys.: Condens. Matter, 2004, 16, L489-L494, doi $10.1088 / 0953-8984 / 16 / 45 / \mathrm{L} 01$

65. Ngai K.L., Wang L.M., Liu R., Wang W.H., J. Chem. Phys., 2014, 140, 044511 (7 pages), doi $10.1063 / 1.4862822$

66. Dyre J.P., Rev. Mod. Phys., 2006, 78, 953-972, doi $10.1103 / R e v M o d P h y s .78 .953$

67. Carbajal-Tinoco M.D., J. Chem Phys., 2008, 128, 184507, doi:10.1063/1.2918495

\title{
Кореляція між кінетичною крихкістю і коефіцієнтом Пуассона на основі аналізу даних для м'яких колоїдів
}

\author{
А. Мондал $1 \frac{11}{1}$ л. Премкумар $p^{112}$, С.П. Дас ${ }^{11}$ \\ 1 Школа фізичних наук, Университет Джавахарлала Неру, Нью-Делі 110067, Індія \\ 2 Фізичний факультет, Національний технологічний інститут Маніпура, Імфал 795004, Індія
}

\begin{abstract}
Ми розглядаємо зв'язок між крихкістю та еластичністю на основі аналізу даних для набору м'яких колоїдних матеріалів що складається з деформованих сфер, описаних в [Mattsson et al., Nature, 2009, 462, 83]. У даній роботі проведено кількісний аналіз з допомогою явного визначення індексу крихкості $m$ в термінах залежності густини, розширюючи відповідну формулу $m$ для молекулярної системи з точки зору температурної залежності. Окрім цього, дані для високочастотного модуля зсуву для відповідного м'якого колоїду застосовано до відповідного теоретичного виразу для того самого модуля. Цей вираз для пружної константи отримано на основі відповідної парної кореляційної функції рідини з однорідною густиною. Парну кореляційну функцію встановлюють шляхом відповідного вибору параметрів для потенціалу взаємодії двох тіл для відповідного матеріалу м'якого колоїду. Природа кореляції між крихкістю і коефіцієнтом Пуассона, що спостерігається для м'яких колоїдів, якісно відрізняється від природи молекулярного шкла. Зв'язок, що спостерігається між крихкістю метастабільної рідини та їі коефіцієнта пружності, $є$ проявом впливу будови рідини на ї̈ динаміку. Таким чином, у даній роботі проаналізовано дані стосовно м'яких колоїдів. За рахунок введення дефініцій зі статистичної механіки, отримано кореляцію між кінетичною крихкістю та коефіцієнтом Пуассона для м'яких матеріалів.
\end{abstract}

Ключові слова: кінетична крихкість, пружний відгук, часи релаксації, склування 


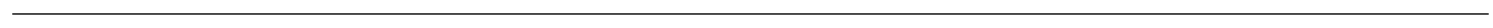

